\title{
PEDF and GDNF are key regulators of photoreceptor development and retinal neurogenesis in reaggregates from chick embryonic retina
}

\author{
Katja N. Volpert • Joyce Tombran-Tink • \\ Colin Barnstable • Paul G. Layer
}

Received: 8 December 2008 / Accepted: 12 January 2009/Published online: 27 January 2009

(C) The Author(s) 2009. This article is published with open access at Springerlink.com

\begin{abstract}
Here, role(s) of pigment epithelial-derived factor (PEDF) and glial-derived neurotrophic factor (GDNF) on photoreceptor development in three-dimensional reaggregates from the retinae of the E6 chick embryo (rosetted spheroids) was investigated. Fully dispersed cells were reaggregated under serum-reduced conditions and supplemented with $50 \mathrm{ng} / \mathrm{ml}$ PEDF alone or in combination with $50 \mathrm{ng} / \mathrm{ml}$ GDNF. The spheroids were analyzed for cell growth, differentiation, and death using proliferating cell nuclear antigen, terminal deoxynucleotidyl transferase deoxyuridine triphosphate nick end labeling, and other immunocytochemical stainings and semi-quantitative reverse transcription polymerase chain reaction (RT-PCR) methods. PEDF strongly promoted synthesis of the messenger RNAs for blue and violet cone opsins and to a lesser extent on the red and green cone opsins. This correlated with an increase in the number of cone photoreceptors, as
\end{abstract}

K. N. Volpert · P. G. Layer

Institut für Zoologie, Technische Universität Darmstadt,

Schnittspahnstrasse 13,

64287 Darmstadt, Germany

J. Tombran-Tink

Department of Ophthalmology, Yale University,

New Haven, CT, USA

C. Barnstable

Department of Neural and Behavioral Sciences,

Penn State College of Medicine,

Hershey, PA 17033, USA

P. G. Layer $(\bowtie)$

Entwicklungsbiologie und Neurogenetik,

Technische Universität Darmstadt,

Schnittspahnstraße 13,

64287 Darmstadt, Germany

e-mail: layer@bio.tu-darmstadt.de determined by the cone cell marker CERN906. Likewise, PEDF nearly completely inhibited rod differentiation, as detected by immunostaining with anti-rho4D2 and RTPCR. Furthermore, PEDF accelerated proliferation of cells in the spheroids and inhibited apoptosis. As negative effects, PEDF inhibited the normal histotypic tissue formation of retinal aggregates and reduced the frequency of photoreceptor rosettes and IPL-like areas. Noticeably, supplementation of PEDF-treated cultures with GDNF reversed the effects of PEDF on spheroid morphology and on rod differentiation. This study establishes that PEDF strongly affects three-dimensional retinogenesis in vitro, most notably by inhibiting rod development and supporting proliferation and differentiation of cones, effects which are partially counteracted by GDNF.

Keywords Cytokines · Muller glial cells · Photoreceptor degeneration · Retinal spheroids · Tissue formation

\section{Introduction}

During retinogenesis, the neural retina develops from the inner layer of the optic vesicle and the retinal pigment epithelium (RPE) from the outer layer. The close proximity of both tissues and their intricate interactions are essential for function, structure, and differentiation of photoreceptors [1-4]. In particular, the development of the photoreceptor outer segments is highly dependent on the RPE, e.g., after RPE removal, the structural organization of the outer segment is disrupted [5]. The interdependence between the RPE and photoreceptors is mediated by the exchange of nutrients, metabolites, and various signal molecules secreted from both cell populations. Numerous growth factors have been identified as RPE-secreted products [6-10], 
including pigment epithelium-derived factor (PEDF) [11]. Analysis of the specific roles of these molecules in vivo is complicated because of their multiple and combined effects ("cocktail effects") on the development, survival, and differentiation of the retina. A previously developed threedimensional in vitro culture of reaggregated retinal organotypic spheres has been found to represent a most suitable model to dissect the effects of individual growth factors in early retinal development. The reaggregates are derived from dissociated cells isolated from 6-day-old chick retina. Under rotation conditions, the cultures will form threedimensional rosetted spheroids that attempt to imitate normal retinal development (see Fig. 3). The innermost part of the spheroids contains rosettes that are composed of photoreceptors and thus correspond to the outer nuclear layer of a normal retina. Each rosette is surrounded by a circular outer plexiform layer, followed by sections of an inner nuclear layer (INL). Next to the INL are inner plexiform layer (IPL)-like areas which are cell-free islandshaped areas, consisting of neurites of bipolar, ganglion, and amacrine cells (for review [12]). Within these IPLs, only a few displaced ganglion and amacrine cells are detectable. In addition to the above structures, there are non-organized areas of the spheroids consisting of different retinal cell types and their corresponding fibers. The rosetted spheroids arise first through aggregation of the dissociated retinal cells, followed by cell proliferation, cell migration, differentiation, and programmed cell death. The spheroids are easy to manipulate and their growth can be altered by supplementation with individual growth factor(s) and a cocktail of molecules or by blocking the expression of key proteins using gene knockdown strategies.

Using this culture model, the function of glial-derived neurotrophic factor (GDNF) on retinal development was investigated [13, 14]. GDNF, a distant member of the transforming growth factor beta superfamily, is a potent neurotrophic factor for a variety of central and peripheral neurons $[15,16]$. Using retinal spheroids, GDNF was shown to regulate proliferation, differentiation, and survival of chicken rod photoreceptors in vitro [13], while it had minor effects on cone photoreceptors. Interestingly, knockdown of a particular receptor for GDNF predominantly affected the development of violet photoreceptors [17]. In particular, GDNF increased proliferation of rod precursors, promoted the onset of their differentiation, increased their number, and prevented programmed cell death.

Here, we focused on another potent factor, PEDF, which is synthesized and secreted by the RPE. Originally, PEDF was isolated from conditioned medium of human RPE cells, inducing neuronal differentiation of human retinoblastoma cells $[11,18]$. The $50-\mathrm{kDa}$ PEDF protein belongs to the family of serine protease inhibitors, however, not exerting proteolytic activity [19]. Being expressed in several parts of the central nervous system and eye tissues of humans [20, 21], PEDF has been assigned several physiological roles in various tissues, e.g., an anti-angiogenic effect during neovascularization [22], including mammalian eyes [23, 24]. In central nervous system and peripheral nervous system neurons from rodent and human origins, PEDF acted as survival factor in vitro [25-27]. The significance of PEDF in the developing neural retina is not well understood. PEDF is expressed by more than one retinal cell type in several mammalian retinas, e.g., Muller and ganglion cells in rodents can express and secrete PEDF $[24,28]$ and possibly protect themselves by this factor [29]. An expression of PEDF in photoreceptors has been detected only in human retinae [24]. Because of its abundant secretion by the RPE, several studies have focused on roles of PEDF for photoreceptor survival. For example, PEDF was capable of protecting rat photoreceptors from death in vitro induced by light, oxidative stress, or glutamateinduced toxicity $[5,30]$.

In the first part of this study, we applied PEDF to rosetted spheroids of retinal reaggregates to investigate its role in proliferation, differentiation, and survival of specific retinal cell types, in particular the photoreceptors. We showed that, while the overall morphologic tissue development remained inferior to the control after treatment, PEDF increased and sustained proliferation and strongly decreased the number of apoptotic cells. Furthermore, PEDF showed opposing effects to GDNF on the differentiation of rod and cone photoreceptors. A combination treatment with both PEDF and GDNF resulted in synergistic effects on the survival and proliferation of cone photoreceptors and the morphology of the spheres. From these studies, we propose that PEDF and GDNF are key players in the development of rod and cone photoreceptors during retinogenesis.

\section{Materials and methods}

\section{Tissue culture}

To produce rosetted retinal spheroids, the retinas from 6day-old chicken embryos (E6, white leghorn) were used. The central parts of the retina were isolated and collected in F12 medium on ice. The retinal tissue was dissociated by tryptic digestion in F12 medium containing $0.05 \mathrm{mg} / \mathrm{ml}$ trypsin (Worthington Biochemicals/Cell Systems, Remagen, Germany) for $8 \mathrm{~min}$ at $37^{\circ} \mathrm{C}$. The remaining cell clusters were mechanically dissociated in Hanks' balanced salt solution containing $0.5 \mathrm{mg} / \mathrm{ml}$ DNase I (Worthington Biochemicals/Cell systems, Remagen, Germany) by 30 to 35 gentle strokes with a round-bored Pasteur pipette. For generation of retinal spheroids, $2 \times 10^{6}$ cells per milliliter were cultured in $35-\mathrm{mm}$ dishes containing 2 -ml aggregation 
medium (Dulbecco's modified Eagle's medium, 2\% fetal calf serum, $1 \%$ L-glutamine, and $0.15 \%$ penicillin/streptomycin, all from Gibco, Berlin, Germany) on a gyratory shaker in an incubator at $37^{\circ} \mathrm{C}$ and $5 \% \mathrm{CO}_{2}$ (Heraeus Holding $\mathrm{GmbH}$, Hanau, Germany), either in absence of growth factors or presence of $50 \mathrm{ng} / \mathrm{ml}$ PEDF, presence of $50 \mathrm{ng} / \mathrm{ml}$ GDNF (Sigma, Deisenhofen, Germany), or in a combination of both.

\section{Cryosections and immunostaining procedures}

For obtaining cryosections, rosetted spheroids were harvested at the indicated time points and fixed in $4 \%$ formaldehyde (Merck, Darmstadt, Germany) for $30 \mathrm{~min}$ at room temperature. After the fixative was removed by two washes in phosphate-buffered saline (PBS), rosetted spheroids were soaked in 25\% sucrose (Merck, Darmstadt, Germany) and stored at $4^{\circ} \mathrm{C}$. Cryosections of $10-\mu \mathrm{m}$ thicknesses were cut on a cryostat (Microm, Waldorf, Germany), mounted on gelatine-coated slides, and stored at $-20^{\circ} \mathrm{C}$.

For immunostaining, sections were dried at $37^{\circ} \mathrm{C}$ and pre-incubated in blocking solution, containing $3 \%$ bovine serum albumin, $0.1 \%$ Triton-X-100 (all from Sigma, Deisenhofen, Germany) in PBS for $30 \mathrm{~min}$ at room temperature. Then, tissues were incubated with the primary antibody for $75 \mathrm{~min}$, followed by three washes in PBS. The rod-specific monoclonal antibody rho4D2 (a generous gift from D. Hicks, Strasbourg, France) was used at a dilution of 1:1,000 in blocking solution, and the red- and greenspecific antibody CERN906 (a generous gift of W. DeGrip, University of Nijmegen, The Netherlands) was used at a dilution of 1:500 in blocking solution. For detection of the primary antibody, sections were incubated with donkey anti-mouse-conjugated $\mathrm{Cy} 3$ and goat anti-rabbit-conjugatedCy2 (both $10 \mu \mathrm{g} / \mathrm{ml}$; Dianova, Hamburg, Germany) for $1 \mathrm{~h}$. For detection of proliferation, the staining reagent proliferating cell nuclear antigen (PCNA) was used at a dilution of 1:250 in blocking solution; cryosections were incubated for $3 \mathrm{~h}$ with PCNA. Before pre-incubating in blocking solution, sections were incubated with $4 \mathrm{~N} \mathrm{HCl}$ for $10 \mathrm{~min}$. For detection of apoptosis, sections were terminal deoxynucleotidyl transferase deoxyuridine triphosphate nick end labeling (TUNEL)-stained according to the manufacturer's instructions (Roche Molecular Biochemicals, Mannheim, Germany). Between the last washes, cell nuclei were stained with 4',6-diamidino-2-phenylindole (DAPI; $0.1 \mathrm{mg} / \mathrm{ml} \mathrm{4',6-diamidine-2-phenylindol-dihydrochloride}$ in PBS; Merck, Darmstadt, Germany). Finally, sections were dried and embedded in Kaiser's glycerine gelatine (Merck, Darmstadt, Germany). It should be noted that, due to limited availability of spheroids, cryosections have been taken not only from their equatorial level. Therefore, different aerial sizes of cryosections as shown in figures do not necessarily indicate a smaller spheroid volume.

\section{RT-PCR}

For semi-quantitative reverse transcription polymerase chain reaction (RT-PCR), total RNA was isolated from retinal cultures at appropriate stages and transcribed into complementary DNA (cDNA) by using the reverse transcription system from Promega (Mannheim, Germany). Target cDNA was amplified by PCR with specific primers. PCR was performed using parameters of $94^{\circ} \mathrm{C}$ for $1 \mathrm{~min}, 57^{\circ} \mathrm{C}$ for $1 \mathrm{~min}$, and $72^{\circ} \mathrm{C}$ for $1 \mathrm{~min}$ for 29 cycles. Primers for PCR were 5'-GGACAGTTCAAGGGCACTGT-3' and 5'CTTCTGTGTGGCTGTGATGG-3' for GAPDH, 5'-TCACC ATCCAGCACAAGAAA-3' and 5'-GACCAGGTTCCCAT AGCAGA-3' for rhodopsin, 5'-ATCAACCAGATCTCG GGGTA- ${ }^{\prime}$ ' and 5'-CAGAAGCAGTAGGCCACG AT-3' for red opsin, 5'-CATGATGGGGATAGCTTTCA- ${ }^{\prime}$ ' and $5^{\prime}$-TGG TGATCATGCAATTACGG-3' for green opsin, $5^{\prime}$-CGCTCT GAGTGTGATGAGGA-3' and 5'-AGCTCCGTTCCC ACCTTAAT- $3^{\prime}$ for blue opsin, and 5'-CGTGGGCCTTCTA CCTACAG-3' $3^{\prime}$ and 5'-TACTCGCT GCGGTATTTCGT-3' for violet opsin. The relative expression of messenger RNA (mRNA) was quantified as a percentage of GAPDH band intensity at the indicated stages.

\section{Cell counting and statistical analysis}

To determine the number of immunostained cells, frozen sections were stained with DAPI and rho4D2 antibody. Then, the percentage of immuno-labeled cells per section of a single spheroid was calculated in relation to DAPI-positive cells of the same spheroid. At least six cryosections of different spheroids derived from two individual experiments were analyzed. Data are presented as the mean $\pm \mathrm{SD}$ and compared by a two-tailed Student's $t$ test. Note that the standard deviation represents the difference between calculations of individual spheroids.

To determine the relative expression of mRNA, the percentage was calculated in relation to GAPDH band intensity for each culture stage. Each experiment was carried out at least three times.

\section{Microscopy and photography}

Photomicrographs of sections were taken with an Axiophot microscope (Carl Zeiss, Jena, Germany) combined with a charge-coupled device three-color (CCD-3) digital camera (Intas, Göttingen, Germany). Photomicrographs were processed on a computer with documentation and analysis software (Diskus Histologie MAN F70B, Hilgers, Königswinter, Germany; Multianalysts 2.0, Biorad, München, 
Germany; Adobe Photoshop CS, Adobe, San Jose, CA, USA; and Excel, Microsoft, Redmond, WA, USA).

\section{Results}

\section{PEDF enhances proliferation}

When PEDF, at a concentration of $50 \mathrm{ng} / \mathrm{ml}$, was added to rosetted spheroids developing from E6 chick retinae under serum-reduced culture conditions, the number of proliferating cells $\left(\mathrm{PCNA}^{+}\right.$cells) was significantly increased (Fig. 1). This proliferative stimulation was most pronounced at early culture stages. At day in vitro (div) 2, $28 \%$ of cells in controls were proliferating, while in PEDFtreated cultures $38 \%$ proliferating cells could be identified (Fig. 1e). The stimulatory effect on proliferation became much more pronounced at days 4 and 6. Compared to controls, the number of proliferating cells was now more than doubled after PEDF treatment. Thus, in the presence of PEDF, $17 \%$ of all cells were proliferating at day 4 and $8 \%$ at day 6 , compared with $6 \%$ and $3 \%$ in the respective controls. At day 8, proliferation had become very low in both cultures and did not differ significantly between treated and non-treated cultures (Fig. 1e).

\section{Apoptosis is strongly decreased by PEDF}

Using the TUNEL assay, a very dramatic inhibitory effect of $50 \mathrm{ng} / \mathrm{ml}$ PEDF on programmed cell death was revealed in our retinal cell cultures (Fig. 2). Histologically, cells of the entire spheroid were evenly protected from apoptosis (cf. Fig. 2b, d). The percentage of cells undergoing apoptosis in PEDF-treated and non-treated cultures was quantified in relation to the total number of DAPI-positive cells at day 10 (Fig. 2e). The percentage of apoptotic cells was only $8 \%$ in presence of PEDF, while in controls $32 \%$ of cells were identified as apoptotic.

PEDF inhibits spatial organization of retinal spheroids

The morphology of spheroids as characterized by typical structures like rosettes and IPL-like areas was also strongly affected (Fig. 3; see also Figs. 1 and 2). In Fig. 3a-c, we show a triple-stained retinal spheroid, stained with DAPI (Fig. 3a), anti-rho4D2 (specific for rod photoreceptors, in Fig. 3b), and anti-CERN906 (specific for red and green cones, in Fig. 3c), with one small photoreceptor rosette (broken circle), composed of rods (Fig. 3b) and cones (Fig. 3c). Near the surface of the sphere, there are four large circular IPL-like areas, each containing a few isolated cells (Fig. 3a, IPL, arrow). In the presence of PEDF, these histotypical structures found in the untreated controls were very rare (Fig. 3d-f; Figs. 1 and 2). Instead, the sphere mostly appeared as a non-organized cell aggregate, suggesting that PEDF strongly interfered with development of the normal tissue organization of the spheroids.

PEDF decreases rho4D2 ${ }^{+}$cells and rhodopsin mRNA expression

Since several neurotrophic factors affect differentiation of various retinal cell types, including photoreceptors, we were interested in the effect of PEDF on rod and cone photoreceptor development in the spheroids (Fig. 3). PEDF strongly decreased the staining for rod photoreceptors as compared to the controls (Fig. 3b, e). In the non-treated controls, groups of rho4D2-positive cells are shown in the center of the rosette as well as at the periphery and in intermediate locations (Fig. 3b). In contrast, spheroids treated with PEDF
Fig. 1 PEDF (50 ng/ml) increases proliferation in retinal spheroids, as identified by PCNA staining. Cryosections of 2-day-old spheroids were double-stained with DAPI (a, c) and with PCNA (b, d). e shows quantification of data. Note that sizes of sections do not reflect true volume size of spheroid (see "Materials and methods"). Each data point represents the mean \pm SD of multiple sections $(n=6) .{ }^{*} * P<0.001 ; * * * P<$ 0.0001. Scale bar, $100 \mu \mathrm{m}$
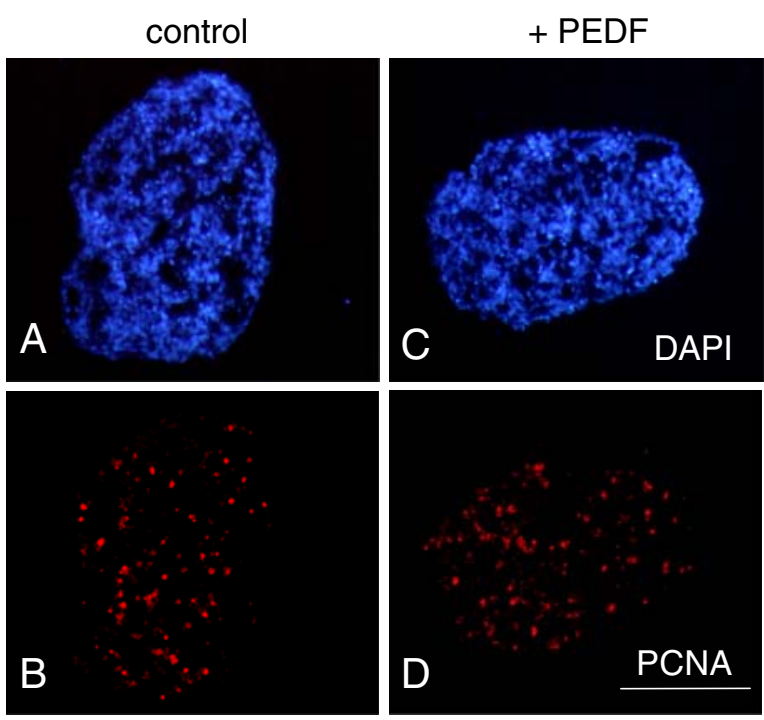
Fig. 2 PEDF (50 ng/ml) strongly inhibits apoptosis in retinal spheroids. Cryosections of 10-day-old spheroids were stained with TUNEL $(\mathbf{b}, \mathbf{d})$. For calculation of the percentage of apoptotic cells, cell nuclei were stained with DAPI $(\mathbf{a}, \mathbf{c})$. The percentage of apoptotic cells was $32 \%$ in controls but only $8 \%$ in PEDF-supplemented cultures (e). Each data point represents the mean $\pm \mathrm{SD}$ of multiple sections $(n=6) . * * P<0.001$. Scale bar, $100 \mu \mathrm{m}$
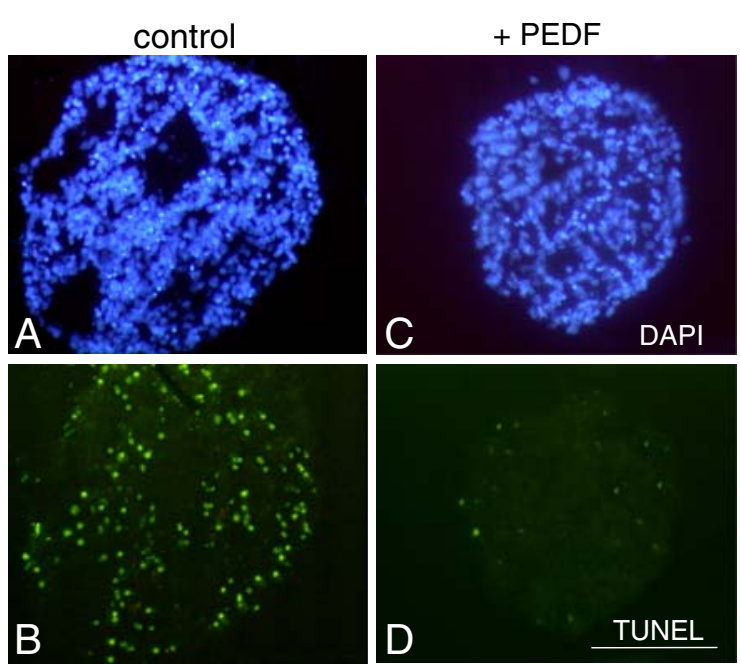

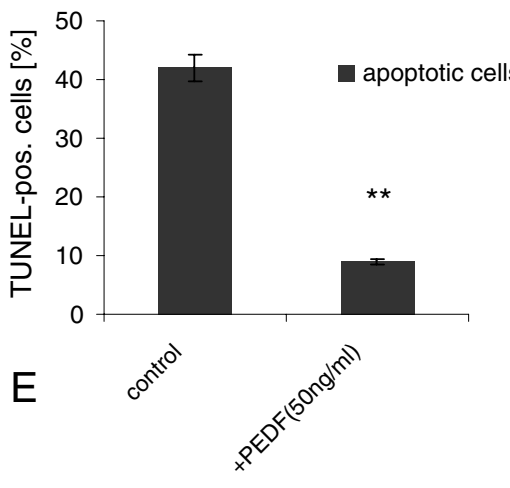

are very weakly stained for rho4D2, with only a few immuno-positive cells present (Fig. 3e). Based on DAPI staining of the identical control and experimental spheroids (Fig. 3a, d), the percentages of rho4D2-positive cells were quantified in controls and in PEDF-treated cultures and the quantitative data presented in Fig. 3g. Between days 8 and 10 , the number of rods in controls accounted for $7.3 \%$ and $5.5 \%$ of the cells, respectively, while, in PEDF-treated cultures, the number of rods represented only about $2.5 \%$ and $2 \%$ of the cells, respectively, accounting for only about one third of the number in the control.

These findings were independently confirmed by semiquantitative RT-PCR (Fig. 4). The temporal expression of endogenous rhodopsin mRNA was determined between days 2 and 10 in controls (Fig. 4a) and in PEDF-treated cultures (Fig. $4 \mathrm{~b}$ ). The percentage of rhodopsin mRNA was calculated relative to the expression of GAPDH mRNA and the quantitative analysis is presented in Fig. 4c. In the absence of PEDF, there was a gradual increase in rhodopsin mRNA from $7 \%$ to $22 \%$ in the cultures at days 2 to 6 with peak levels at day 6 . This was followed by a decrease to about $3 \%$ by day 10 . In the presence of PEDF, a quantitatively and temporally different expression pattern was revealed over this time in culture. Rhodopsin expression showed a very high level of $24 \%$ already at day 4 . But then, rhodopsin expression was quickly and efficiently downregulated, leading to a sharp decline to $0.2 \%$ by day 10. These studies indicate that PEDF accelerated
Fig. 3 PEDF $(50 \mathrm{ng} / \mathrm{ml})$ decreases general spheroid tissue differentiation (shown by DAPI in a, d) and differentiation of rod photoreceptors $(\mathbf{b}, \mathbf{e})$ and increases number of cone photoreceptors (c, f). The IPL-like areas (ipl in a) and rosettes (ros, broken circles in $\mathbf{a}-\mathbf{c})$ are absent in PEDF-treated spheroids. Cryosections of 8-day-old spheroids were triple-stained with the rodspecific antibody rho4D2 (red; b, e) and the red and green conespecific antibody CERN-906 (green; c, f). The percentage of rho4D2- and CERN906-positive cells was calculated in relation to DAPI-positive cells (g, h). Each data point represents the mean $\pm \mathrm{SD}$ of multiple sections $(n=6) .{ }^{*} * P<0.001 ; * * * P<$ 0.0001. Scale bar, $100 \mu \mathrm{m}$
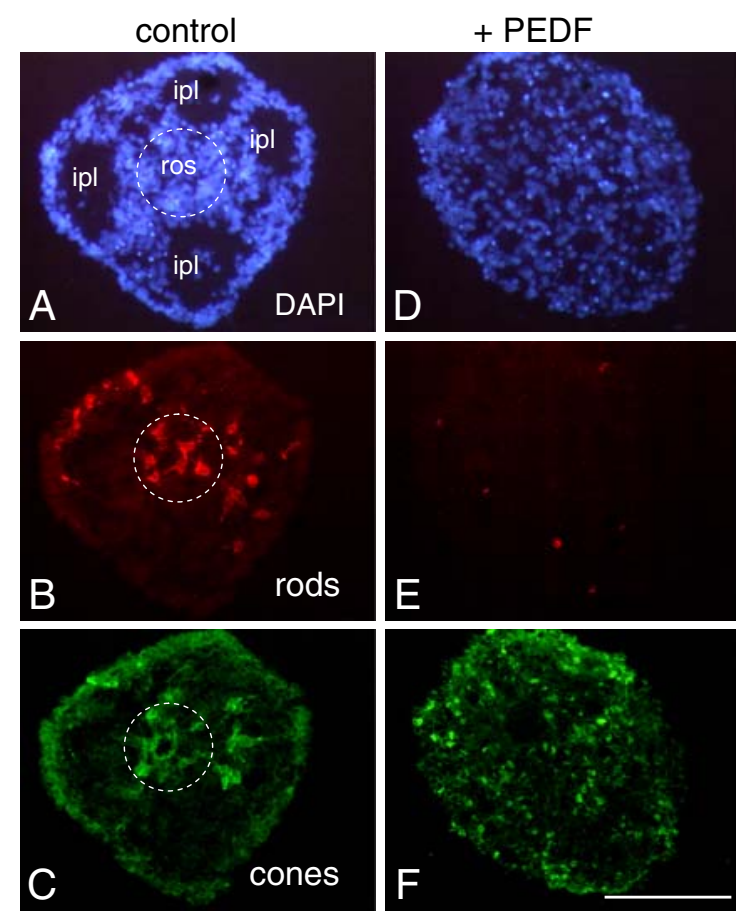

G
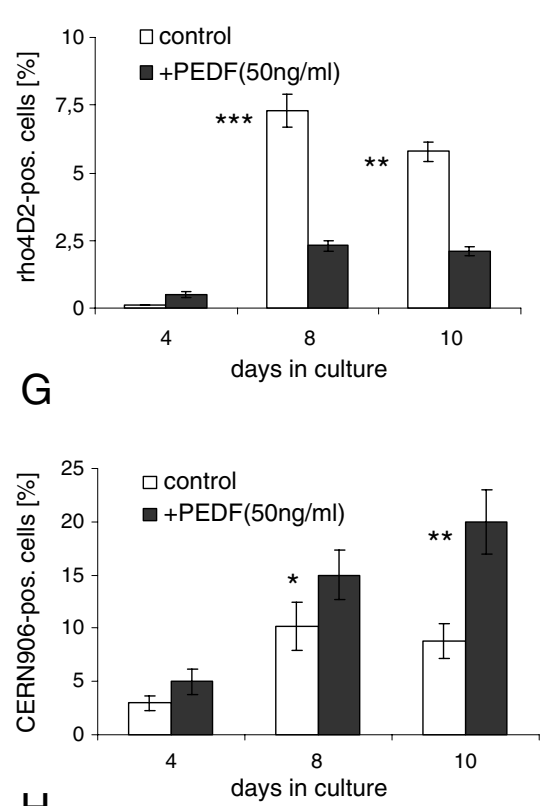

$\mathrm{H}$ days in culture 
Fig. 4 Temporal expression of rhodopsin mRNA in rosetted spheroids (a) or in the presence of $50 \mathrm{ng} / \mathrm{ml}$ PEDF (b), as analyzed by semi-quantitative RT-PCR at different culture days; the percentage of rhodop$\sin$ mRNA was calculated in relation to expression of GAPDH mRNA (c). Note the strong decrease of rhodopsin mRNA in the presence of PEDF (further see text). ${ }^{* * P} P<0.001$

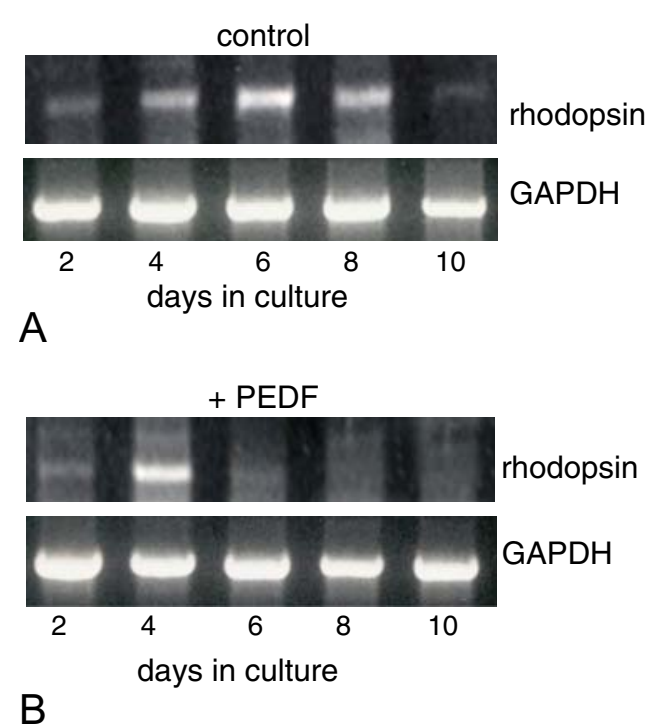

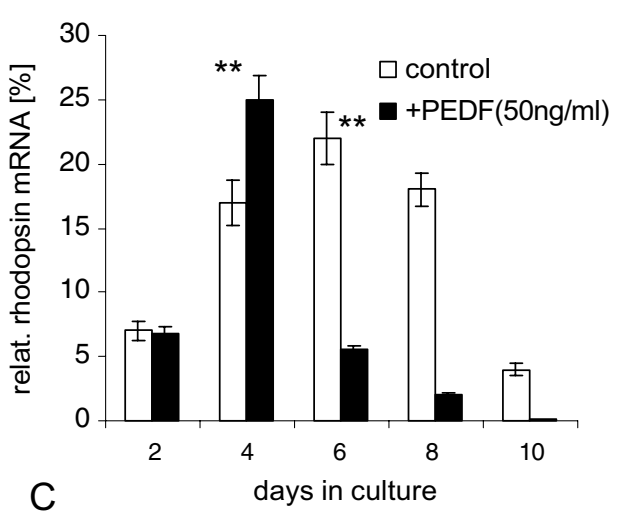

rhodopsin expression for a short period of time, then almost completely inhibited it.

\section{PEDF increases CERN906 ${ }^{+}$cones}

While PEDF showed dramatic inhibitory effects on rho4D2-positive cells and on rhodopsin mRNA expression in the spheroids, the number of CERN906-positive cone photoreceptors was significantly increased, as revealed by the red/green cone-specific antibody CERN906 (Fig. 3c, f). Similar to the arrangement of rods, CERN906-positive cells were found inside the rosette, and a relatively high number was also seen at the periphery in control cultures (Fig. 3c). After treatment with PEDF, many CERN906-positive cells were found distributed all over the aggregate (Fig. 3f). At all time points examined, the number of red and green CERN906-positive cone cells was higher in PEDF-treated cultures (Fig. 3h). At day 8, there were 15\% CERN906positive cells in the presence of PEDF and $9 \%$ in the absence of this neurotrophic factor. At day 10, the number of cones represented $20 \%$ and $8 \%$ of the total cell population with and without PEDF treatment, respectively. Note that while, in the control cultures, red and green cone cell number decreased from day 8 to 10 , in the presence of PEDF, the highest number of CERN906-positive cells was observed at day 10, suggesting a long-lasting effect of PEDF on cone cell differentiation and survival.

Subpopulations of cones are selectively affected by PEDF

The effects of PEDF on specific types of opsins were analyzed by semi-quantitative RT-PCR (Fig. 5). We analyzed the temporal expression of RNAs for red, green, blue, and violet cone opsins in the controls (Fig. 5a) and in
PEDF-treated cultures between days 2 and 10 in culture (Fig. 5b). PEDF increased the expression of all four cone opsin RNAs, although each showed a distinctive pattern. The data are quantified and compared in Fig. 6a-d.

The expression of red cone opsin RNA following PEDF treatment showed a rapid increase and then stayed at an almost steady level of approximately $20 \%$, while in controls it decreased after div 6 (Fig. 6a). The expression of green cone opsin was very similar to that of red cone opsin (Fig. 6b vs. a). PEDF induced a rapid increase in expression of blue cone opsin RNA between div 2 and 6 (approx-

Fig. 5 Temporal expression of mRNAs of four cone opsins in rosetted spheroids (a) and after treatment with $50 \mathrm{ng} / \mathrm{ml}$ PEDF (b). Opsin mRNA was analyzed by semi-quantitative RT-PCR at different days in culture

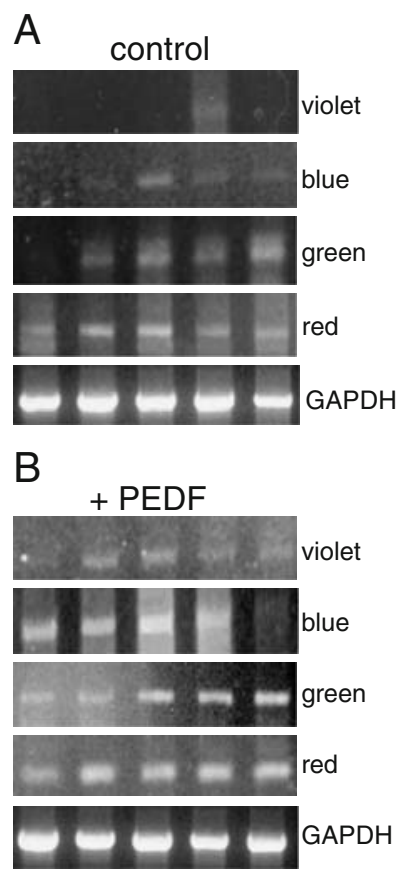


Fig. 6 Quantification of mRNA levels from Fig. 5. The percentage of opsin mRNA was calculated in relation to expression of GAPDH mRNA. All opsins showed higher mRNA expression levels for PEDF-treated cultures. At days 8 and 10, the expression of red opsin remained over $20 \%$, whereas in controls expression decreased to less than $10 \%$ (a). Controls and PEDF-treated cultures showed similar expression patterns for blue and green opsin (b vs. a), but note the different expression intensities for blue and violet opsins (c, d). Both blue and violet opsin mRNAs showed very different expression patterns and higher intensities after PEDF treatment. $* P<0.01$; $* * P<0.001 ; * * * P<0.0001$
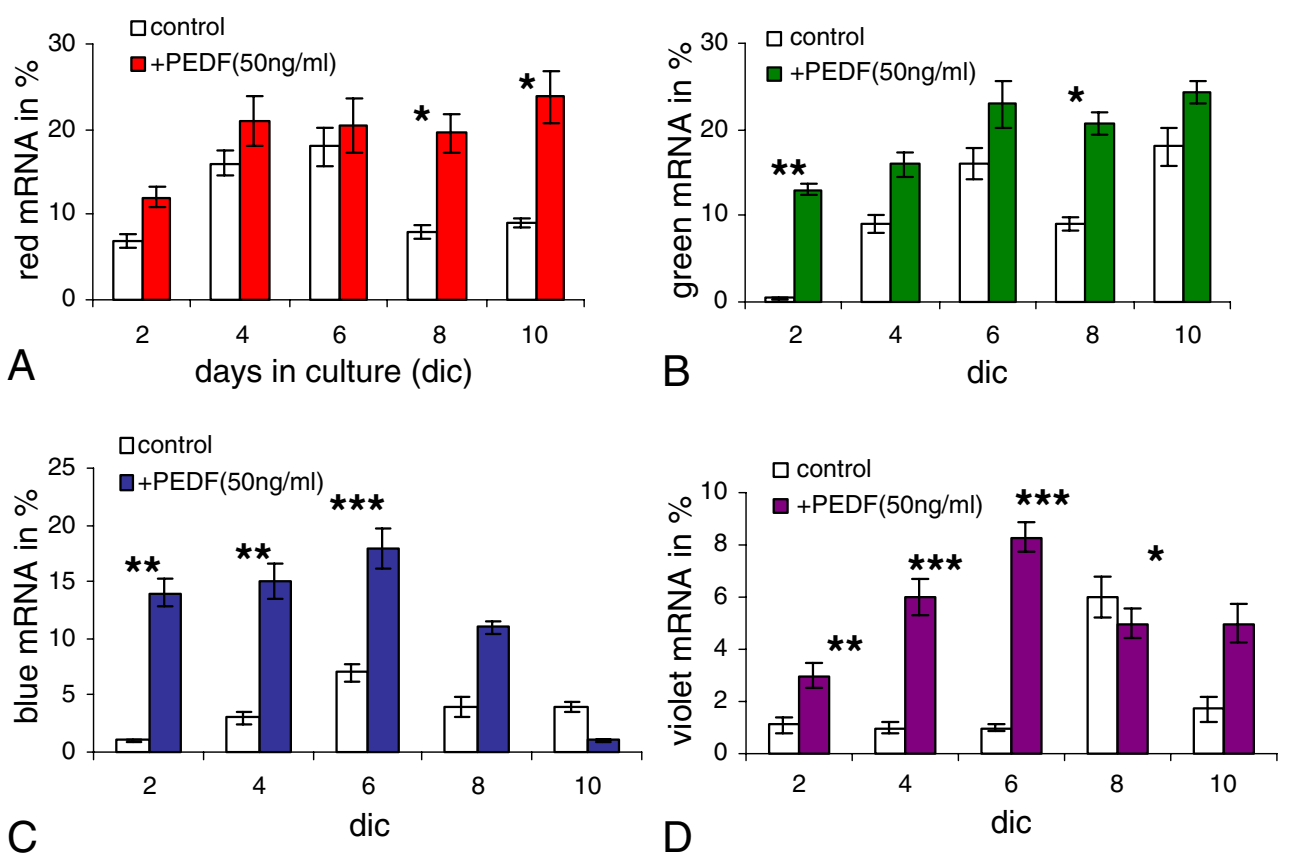

imately sevenfold at div 2), after which time the levels decreased (Fig. 6c). Finally, violet cone opsin expression was influenced by PEDF in a pattern similar to that for blue cone opsin, e.g., at div 6 PEDF led to an approximately sevenfold increase of violet opsin expression (Fig. 6d; for its high expression in the control at div 8, see "Discussion"). In summary, while all four types of cone opsin expression were increased by PEDF, most pronounced effects were documented for blue and violet opsins.

GDNF in combination with PEDF restores the spatial organization of retinal spheroids

Since application of PEDF disrupted the spatial organization of rosetted spheroids (Figs. 1, 2, and 3) and had very different effects to those we have previously reported for GDNF, we were interested in the effects of a combined application of PEDF and GDNF. Application of $50 \mathrm{ng} / \mathrm{ml}$ GDNF plus $50 \mathrm{ng} / \mathrm{ml}$ PEDF completely restored the morphology of rosetted spheroids (Fig. $7 \mathrm{~d}-\mathrm{f}$ ). The number and size of IPL areas appeared normal. Thus, GDNF counteracted the disrupting effects of PEDF on tissue formation of the spheroids.

GDNF counterbalances the effects of PEDF on rod photoreceptors

Since GDNF reversed the effects of PEDF on the morphology of spheres, we investigated the effect of combined treatment on photoreceptor regulation (Fig. 7). For both rods and cones, the combined treatment with
GDNF and PEDF promoted a significant increase as compared with PEDF treatment alone (Fig. 3). While, by day 8 , the percentage of rho4D2-positve cells was still about one third lower than in the control, by day 10 it was nearly doubled (Fig. 7g). The effects of a combined GDNF/ PEDF treatment were at least as significant for CERN906positive cone cells (Fig. 7c, f): at both days 8 and 10, the percentage of red/green cones was approximately doubled (Fig. 7h). Moreover, whereas in controls the percentage of CERN906-positive cells decreased from day 8 to day 10 , in GDNF/PEDF-treated cultures it stayed constant over the remaining culture period at $13 \%$.

\section{Discussion}

We have used a three-dimensional reaggregate model of the embryonic chick retina to investigate effects of PEDF on retinal tissue formation in vitro. Our findings have documented distinct effects of PEDF on the regulation of cell proliferation and apoptosis, as well as on the differentiation of rod and cone photoreceptors. Together, these effects resulted in an altered three-dimensional tissue structure.

PEDF increases spheroid size but inhibits tissue structure

Rosetted spheroids of retinal cells develop a very characteristic histotypic structure (see "Introduction"). Since we raised our cultures here under serum-reduced conditions, this typical structure was not always completely represented 
Fig. 7 Combined application of GDNF and PEDF (both at $50 \mathrm{ng} / \mathrm{ml}$ ) restores number of rods and increases percentage of cones. Cryosections of 10-day-old spheroids were triple-stained with DAPI $(\mathbf{a}, \mathbf{d})$, the rod-specific antibody rho4D2 (red; b, e), and the red and green cone-specific antibody CERN906 (green; c, f). Data are quantified as percentage of DAPI-stained cells in $\mathbf{g}$ and $\mathbf{h}$. Each data point represents the mean \pm SD of multiple sections $(n=6) . * * P<0.001 ; * * P<0.0001$. Scale bar, $100 \mu \mathrm{m}$
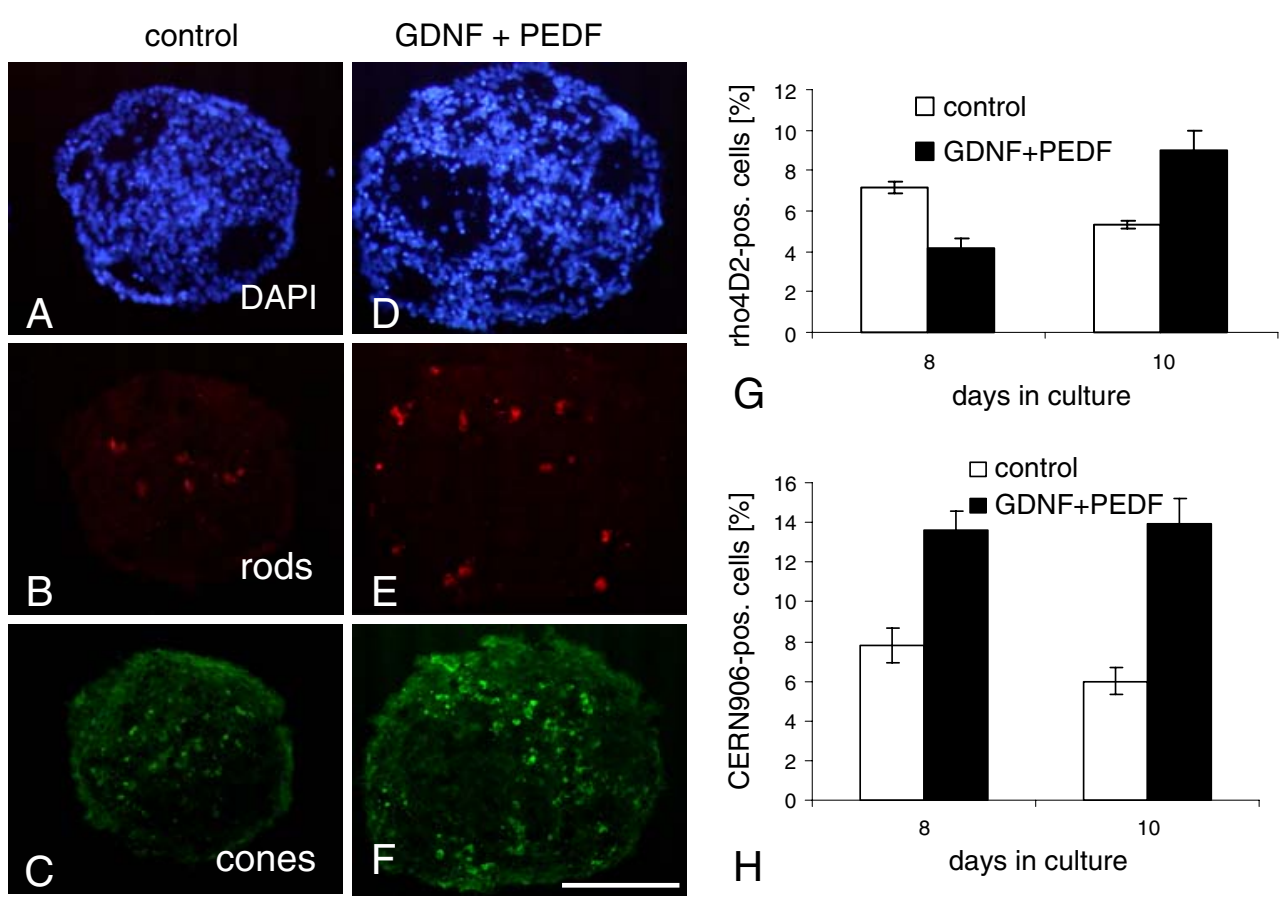

in each and every spheroid. Nevertheless, it was most obvious that the application of exogenous PEDF to cultures led to dramatic morphologic changes in rosetted spheroids. The most obvious difference was a lack of detectable rosettes. In addition, only a few IPL-like areas were formed, and these were small in size and disrupted in organization. At present, we investigate whether PEDF disrupts spheroid morphology by acting on Muller glial cells $[12,31]$ since this histologically essential cell type [32] is affected by several factors [33-36].

\section{PEDF stimulates proliferation and inhibits apoptosis} in rosetted spheroids

Trophic factors can influence diverse developmental processes and regulate cell proliferation and/or cell death of various cell populations in rodents [30, 37, 38]. Mainly studied in mammals, PEDF has been found to have potent anti-proliferative and pro-differentiation effects [39, 40]. However, in our chicken spheroid culture, the application of PEDF induced an increase in cell proliferation, probably due to species differences. Moreover, since proliferation in this model lasts only for a few days, this apparent difference in effect could also be due to an acceleration rather than an increase of proliferation, so that in effect the system would be pushed towards earlier differentiation.

The application of PEDF led to a significant decrease in the number of apoptotic cells; an effect that lasted over extended culture periods. This finding is remarkable since growth factors that prevent programmed cell death foster hopes for the development of therapies for degenerative diseases. Not only could cell death be downregulated in cell culture systems supplemented with neurotrophic factors but also in animal models, in which growth factors were injected into degenerated tissues [41]. Our findings strongly support earlier studies (see below) that PEDF could become a candidate to prevent cells from dying in a blinding disease.

PEDF acts as a pro-differentiating factor for cone photoreceptors

The interaction between the retina and the RPE is essential for the structural and functional integrity of photoreceptors (see "Introduction"), and proteins secreted from the RPE support the survival of photoreceptors [42-44]. One of the most important of these is PEDF. It was shown earlier [5] that, after the application of PEDF, deleterious effects of RPE removal on rod photoreceptor ultrastructure, the composition of outer segment membranes, and the expression of opsins were reversed. Under in vitro conditions, PEDF protected photoreceptors from cell death induced by oxidative stress or light $[30,45,46]$. Also, injection of PEDF in eyes with an inherited retinal degeneration caused a significant protection and delay in the cell death of photoreceptors [47].

The role of PEDF in the developing retina is much less well characterized. PEDF is expressed early in retinal development [24], but its ability to influence retinal cell development has not previously been studied. Using both immunocytochemistry and RT-PCR, we found that PEDF decreased the number of rhodopsin-positive cells in the 
spheroids by as much as two thirds. An early spurt of rhodopsin expression at day 4 i.c., which quickly was downregulated again (Fig. 4c), could indicate that PEDF pushes cells towards differentiation of rods but then reverts them to go into a cone lineage. Since rhodopsin is one of the first cell-type-specific genes expressed by developing rods, the results imply that PEDF is preventing retinal progenitors from adopting a rod phenotype.

On the other hand, PEDF increased the number of differentiated cone photoreceptors. By RT-PCR analyses, we identified stimulatory effects of PEDF on red, green, and much more strongly on blue and violet opsins. Using an antibody that recognized red and green opsin cones to label sections of spheroids, we found that the number of these cells was substantially increased by PEDF. This suggests that the major effect of PEDF is on the formation of differentiated cones and not just on the level of opsin expression by differentiated cells. How can the differential effect of PEDF on rods and cones be explained? Cones are born before rods, both in vivo and in spheroids [48-50]. We suggested earlier that PEDF may be accelerating differentiation of retinal cells. If this is the case, PEDF may be driving cells into earlier formed cell types (cones) leaving a decreased number of progenitors to form later cell types (rods). Applying earlier markers for photoreceptor precursors should further clarify this point.

\section{GDNF reverses the effects of PEDF}

In most in vivo situations, many neurotrophic factors are present and may act simultaneously and in concert (cocktail effects). Different factors can act sequentially, or simultaneously and additively (synergistically), or in a mutually inhibitory fashion [51-53]. It is also possible that several populations of cells need to cooperate in order to achieve combined effects of neurotrophic factors. For instance, subpopulations of developing sensory and motoneurons are dependent on the simultaneous action of GDNF and BDNF [54]. A combination of GDNF and CNTF achieved a higher protection of photoreceptors in several model systems [55]. GDNF and BDNF supported the survival of ganglion cells much more efficiently, as compared with either factor applied separately [56]. Analyzing cocktail effects can be extremely difficult, in particular under in vivo conditions. Retinal spheroids provide a useful three-dimensional tissue environment easily amenable for such studies.

We have previously shown that GDNF strongly supports rod development in spheroids but has minimal effects on cones, an effect opposite to that found for PEDF. Therefore, we applied GDNF together with PEDF to investigate possible interactions of GDNF and PEDF and to test whether one action was dominant. For rods, GDNF effectively reversed the inhibition by PEDF. Noticeably, the number of rods was still lower than in controls at the earlier time tested (div 8) but then was much increased by div 10, supporting again the above assumption that PEDF at earlier time points shifts the rod to cone ratio towards cones (see above). Addition of GDNF did not inhibit the action of PEDF in promoting red- and green cone opsin expression. Thus, a cocktail of the two factors eventually resulted in increased differentiation of both photoreceptor cell types. Overall, the addition of GDNF to PEDF in the spheroid cultures resulted in a restoration of the tissue formation and morphology to that seen in the control.

One possible explanation of the results is that while PEDF promotes differentiation of early progenitors, it could inhibit differentiation of late progenitors. GDNF promotes differentiation in a way that overcomes the inhibitory effect of PEDF. This could be by direct action on the progenitors or by an indirect action through other retinal cells, e.g., Muller glial cells. Muller cells are important for retinal tissue formation and their destruction leads to retinal disorganization in vivo and in vitro [32]. With their morphology reaching through the entire retinal width, Muller cells could well be cellular mediators for the effects of GDNF and/or PEDF on both photoreceptors and cells of the inner retina. For instance, a rescue of photoreceptors by GDNF was mediated via retinal Muller cells, possibly due to upregulating FGF-2, which in turn could act on photoreceptors [57]. This mechanistic scheme puts Muller cells into the center of GDNF actions in the retina and could well represent the cellular locus where the two factors compete with each other.

In summary, we have found four major effects of PEDF on the formation of retinal spheroids. First, it promoted cell proliferation and inhibited apoptosis. Second, it inhibited the overall spatial organization of rosetted spheroids. Third, PEDF inhibited the differentiation of rhodopsin-positive cells. Fourth, PEDF promoted the differentiation of all classes of cones (or their precursors), albeit with different kinetics and efficiencies. While some of the inhibitory effects of PEDF could be (in part) reversed by GDNF, the combination of the two factors resulted in the best differentiation of both classes of photoreceptors (note that differentiation of photoreceptors in spheroids never is complete). The use of neurotrophic factors or combinations of factors suggests fascinating possibilities to develop therapies for degenerating eye diseases, like retinitis pigmentosa [58-60]. A combination of PEDF and GDNF might be one such therapy and should be investigated further.

Acknowledgements We thank F. Frohns, D.M. Gamm, M. Rieke, L.E. Sperling, A. Vogel-Höpker, and E. Willbold for helpful discussions. We acknowledge the expert technical assistance by U. Hoppe, J. Huhn, and M. Stotz-Reimers. This work was supported by the Deutsche Forschungsgemeinschaft (DFG, La 379/12-4). 
Open Access This article is distributed under the terms of the Creative Commons Attribution Noncommercial License which permits any noncommercial use, distribution, and reproduction in any medium, provided the original author(s) and source are credited.

\section{References}

1. Hollyfield JG, Witkovsky P. Pigmented retinal epithelium involvement in photoreceptor development and function. J Exp Zool 1974;189:357-78.

2. Layer PG, Rothermel A, Willbold E. Inductive effects of the retinal pigmented epithelium (RPE) on histogenesis of the avian retina as revealed by retinospheroid technology. Sem Cell Dev Biol 1998;9:257-262.

3. Martínez-Morales JR, Rodrigo I, Bovolenta P. Eye development: a view from the retina pigmented epithelium. Bioessays 2004;26:766-777.

4. Bharti K, Nguyen MT, Skuntz S, et al. The other pigment cell: specification and development of the pigmented epithelium of the vertebrate eye. Pigment Cell Res 2006;19:380-394.

5. Jablonski MM, Tombran-Tink J, Mrazek DA, et al. Pigment epithelium-derived factor supports normal development of photoreceptor neurons and opsin expression after retinal pigment epithelium removal. J Neurosci 2000;20:7149-7157.

6. Hewitt AT, Lindsey JD, Carbott D, et al. Photoreceptor survivalpromoting activity in interphotoreceptor matrix preparations: characterization and partial purification. Exp Eye Res 1990;50:79-88.

7. Campochiaro PA, Sugg R, Grotendorst G, et al. Retinal pigment epithelial cells produce PDGF-like proteins and secrete them into their media. Exp Eye Res 1989;49:217-27.

8. Plouet J, Gospodarowicz D. Transforming growth factor beta-1 positively modulates the bioactivity of fibroblast growth factor on corneal endothelial cells. J Cell Physiol 1989;141:392- 9.

9. Fassio JB, Brockman EB, Jumblatt M, et al. Transforming growth factor alpha and its receptor in neural retina. Invest Ophthalmol Vis Sci 1989;30:1916-22.

10. Connor TB Jr, Roberts AB, Sporn MB, et al. Correlation of fibrosis and transforming growth factor-beta type 2 levels in the eye. J Clin Invest 1989;183:1661-6.

11. Tombran-Tink J, Johnson LV. Neuronal differentiation of retinoblastoma cells induced by medium conditioned by human RPE cells. Invest Ophthalmol Vis Sci 1989;30:1700-7.

12. Layer PG, Rothermel A, Willbold E. From stem cells towards neural layers: a lesson from re-aggregated embryonic retinal cells. NeuroReport 2001;12:A39-46.

13. Rothermel A, Layer PG. GDNF regulates chicken rod photoreceptor development and survival in reaggregated histotypic retinal spheres. Invest Ophthalmol Vis Sci 2003;44:2221-8.

14. Volpert KN, Rothermel A, Layer PG. GDNF stimulates rod photoreceptors and dopaminergic amacrine cells in chicken retinal reaggregates. Invest Ophthalmol Vis Sci 2007;48:5306-14.

15. Lin LF, Doherty DH, Lile JD, et al. GDNF: a glial cell linederived neurotrophic factor for midbrain dopaminergic neurons. Science 1993;21:1130-2.

16. Saarma M, Sariola H. Other neurotrophic factors: glial cell linederived neurotrophic factor (GDNF). Microsc Res Tech 1999;45:292-302.

17. Rothermel A, Volpert K, Burghardt M, et al. Knock-down of GFR $\alpha 4$ expression by RNA interference affects the development of retinal cell types in three-dimensional histiotypic retinal spheres. Invest Ophthalmol Vis Sci 2006;47:2716-25.

18. Tombran-Tink J, Chader GG, Johnson LV. PEDF: a pigment epithelium-derived factor with potent neuronal differentiative activity. Exp Eye Res 1991;53:411-4.
19. Becerra SP, Palmer I, Kumar A, et al. Overexpression of fetal human pigment epithelium-derived factor in Escherichia coli. A functionally active neurotrophic factor. J Biol Chem 1993; 268:23148-56.

20. Ortego J, Escribano J, Crabb J, et al. Identification of a neuropeptide and neuropeptide-processing enzymes in aqueous humor confers neuroendocrine features to the human ocular ciliary epithelium. J Neurochem 1996;66:787-96.

21. Tombran-Tink J, Mazuruk K, Rodriguez IR, et al. Organization, evolutionary conservation, expression and unusual Alu density of the human gene for pigment epithelium-derived factor, a unique neurotrophic serpin. Mol Vis 1996;2:11.

22. Tombran-Tink J, Barnstable CJ. Therapeutic prospects for PEDF: more than a promising angiogenesis inhibitor. Trends Mol Med 2003;9:244-50.

23. Dawson DW, Volpert OV, Gillis P, et al. Pigment epitheliumderived factor: a potent inhibitor of angiogenesis. Science 1999;285:245-8.

24. Behling KC, Surace EM, Bennett J. Pigment epithelium-derived factor expression in the developing mouse eye. Mol Vis 2002;8:449-54.

25. Araki T, Taniwaki T, Becerra SP, et al. Pigment epitheliumderived factor (PEDF) differentially protects immature but not mature cerebellar granule cells against apoptotic cell death. J Neurosci Res 1998;53:7-15.

26. Bilak MM, Corse AM, Bilak SR, et al. Pigment epitheliumderived factor (PEDF) protects motor neurons from chronic glutamate-mediated neurodegeneration. J Neuropathol Exp Neurol 1999;58:719-28.

27. DeCoster MA, Schabelman E, Tombran-Tink J, et al. Neuroprotection by pigment epithelial-derived factor against glutamate toxicity in developing primary hippocampal neurons. J Neurosci Res 1999;56:604-10.

28. Eichler W, Yafai Y, Keller T, et al. PEDF derived from glial Muller cells: a possible regulator of retinal angiogenesis. Exp Cell Res 2004;299:68-78.

29. Li H, Tran VV, Hu Y, et al. A PEDF N-terminal peptide protects the retina from ischemic injury when delivered in PLGA nanospheres. Exp Eye Res 2006;83:824-33.

30. Cao W, Tombran-Tink J, Elias R, et al. In vivo protection of photoreceptors from light damage by pigment epithelium-derived factor. Invest Ophthalmol Vis Sci 2001;42:1646-52.

31. Willbold E, Layer PG. Muller glia cells and their possible roles during retina differentiation in vivo and in vitro. Histol Histopathol 1998;13:531-52.

32. Willbold E, Reinicke M, Lance-Jones C, et al. Muller glia stabilizes cell columns during retinal development: lateral migration but not neuropil growth is inhibited in mixed chick-quail retinospheroids. Eur J Neurosci 1995;7:2277-84.

33. Kirsch M, Lee MY, Meyer V, et al. Evidence for multiple, local functions of ciliary neurotrophic factor (CNTF) in retinal development: expression of CNTF and its receptors and in vitro effects on target cells. J Neurochem 1997;68:979-90.

34. Ju WK, Lee MY, Hofmann HD, et al. Expression of CNTF in Muller cells of the rat retina after pressure-induced ischemia. Neuroreport 1999;10:419-22.

35. Sagar SM, Edwards RH, Sharp FR. Epidermal growth factor and transforming growth factor alpha induce c-fos gene expression in retinal Muller cells in vivo. J Neurosci Res 1991;29:549-59.

36. Roque RS, Caldwell RB, Behzadian MA. Cultured Muller cells have high levels of epidermal growth factor receptors. Invest Ophthalmol Vis Sci 1992;33:2587-95.

37. Steinberg RH. Survival factors in retinal degenerations. Curr Opin Neurobiol 1994;4:515-24.

38. Faktorovich EG, Steinberg RH, Yasumura D, et al. Basic fibroblast growth factor and local injury protect photoreceptors from light damage in the rat. J Neurosci 1992;12:3554-67. 
39. Fernandez-Garcia NI, Volpert OV, Jimenez B. Pigment epithelium-derived factor as a multifunctional antitumor factor. J Mol Med 2007;85:15-22.

40. Ek ET, Dass CR, Choong PF. Pigment epithelium-derived factor: a multimodal tumor inhibitor. Mol Cancer Ther 2006;5:1641-6.

41. Azadi S, Johnson LE, Paquet-Durand F, et al. CNTF + BDNF treatment and neuroprotective pathways in the rd1 mouse retina. Brain Res 2007;1129:116-29.

42. Gaur VP, Liu Y, Turner JE. RPE conditioned medium stimulates photoreceptor cell survival, neurite outgrowth and differentiation in vitro. Exp Eye Res 1992;54:645-59.

43. Sheedlo HJ, Li L, Gaur VP, et al. Photoreceptor rescue in the dystrophic retina by transplantation of retinal pigment epithelium. Int Rev Cytol 1992;138:1-49.

44. Sheedlo HJ, Nelson TH, Lin N, et al. RPE secreted proteins and antibody influence photoreceptor cell survival and maturation. Brain Res Dev Brain Res 1998;107:57-69.

45. Cao W, Tombran-Tink J, Chen W, et al. Pigment epitheliumderived factor protects cultured retinal neurons against hydrogen peroxide-induced cell death. J Neurosci Res 1999;57:789-800.

46. Imai D, Yoneya S, Gehlbach PL, et al. Intraocular gene transfer of pigment epithelium-derived factor rescues photoreceptors from light-induced cell death. J Cell Physiol 2005;202:570-8.

47. Cayouette M, Smith SB, Becerra SP, et al. Pigment epitheliumderived factor delays the death of photoreceptors in mouse models of inherited retinal degenerations. Neurobiol Dis 1999;6:523-32.

48. Prada C, Puga J, Pérez-Méndez L, et al. Spatial and temporal patterns of neurogenesis in the chick retina. Eur J Neurosci 1991;3:1187.

49. Bruhn SL, Cepko CL. Development of the pattern of photoreceptors in the chick retina. J Neurosci 1996;16:1430-9.

50. Rothermel A, Layer PG. Photoreceptor plasticity in reaggregates of embryonic chick retina: rods depend on proximal cones and on tissue organization. Eur J Neurosci 2001;13:949-58.
51. Buchman VL, Davies AM. Different neurotrophins are expressed and act in a developmental sequence to promote the survival of embryonic sensory neurons. Development 1993;118:989-1001.

52. Molliver DC, Snider WD. Nerve growth factor receptor TrkA is down-regulated during postnatal development by a subset of dorsal root ganglion neurons. J Comp Neurol 1997;381:428-38.

53. Hashino E, Johnson EM Jr, Milbrandt J, et al. Multiple actions of neurturin correlate with spatiotemporal patterns of Ret expression in developing chick cranial ganglion neurons. J Neurosci 1999;19:8476-86.

54. Erickson JT, Brosenitsch TA, Katz DM. Brain-derived neurotrophic factor and glial cell line-derived neurotrophic factor are required simultaneously for survival of dopaminergic primary sensory neurons in vivo. J Neurosci 2001;21:581-9.

55. Ogilvie JM, Speck JD, Lett JM. Growth factors in combination, but not individually, rescue rd mouse photoreceptors in organ culture. Exp Neurol 2000;161:676-85.

56. Koeberle PD, Ball AK. Neurturin enhances the survival of axotomized retinal ganglion cells in vivo: combined effects with glial cell line-derived neurotrophic factor and brain-derived neurotrophic factor. Neuroscience 2002;110:555-67.

57. Hauck SM, Kink1 N, Deeg CA, et al. GDNF family ligands trigger indirect neuroprotective signaling in retinal glial cells. Mol Cell Biol 2006;26:2746-57.

58. Jomary C, Vincent KA, Grist J, et al. Rescue of photoreceptor function by AAV-mediated gene transfer in a mouse model of inherited retinal degeneration. Gene Ther 1997;4:683-90.

59. Liang FQ, Aleman TS, Dejneka NS, et al. Long-term protection of retinal structure but not function using RAAV.CNTF in animal models of retinitis pigmentosa. Mol Ther 2001;4:461-72.

60. Martin KR, Quigley HA, Zack DJ, et al. Gene therapy with brainderived neurotrophic factor as a protection: retinal ganglion cells in a rat glaucoma model. Invest Ophthalmol Vis Sci 2003;44:4357-65. 